\title{
Genome organization and transactivation of the human immunodeficiency virus type 2
}

\author{
Mireille Guyader, Michael Emerman, Pierre Sonigo, François Clavel*, Luc Montagnier \\ \& Marc Alizon ${ }^{\dagger}$
}

Unité d'Oncologie Virale (CNRS UA 1157), and Unité de Recombinaison et Expression Génétique (INSERM U163, CNRS UA271), Laboratoire de Biologie Moléculaire et Immunologie des Rétrovirus, Institut Pasteur, 25 rue du Dr Roux 75724 Paris Cedex 15, France

\begin{abstract}
Analysis of the nucleotide sequence of the human retrovirus associated with AIDS in West Africa, HIV-2, shows that it is evolutionarily distant from the previously characterized HIV-1. We suggest that these viruses existed long before the current AIDS epidemics. Their biological properties are conserved in spite of limited sequence homology; this may help the determination of the structure-function relationships of the different viral elements.
\end{abstract}

THE acquired immune deficiency syndrome (AIDS) has now spread worldwide and appears to be an acute public health problem in Africa in particular ${ }^{1-5}$. A retrovirus designated human immunodeficiency virus (HIV), but previously known as LAV, HTLV-III or ARV, was shown to cause AIDS in the different areas afflicted by the epidemics ${ }^{6-8}$. Indeed, isolates from North America, Western Europe and Central Africa have the same biological properties, and antigenically cross-reactive proteins with the same relative molecular mass ${ }^{9-11}$. Only studies at the molecular level have revealed some differences in the nucleotide sequence of North-American and African isolates $^{12,13}$. This sequence variation is also present, though to a lesser extent, among different isolates from the USA ${ }^{14-18}$.

The western part of Africa seemed relatively spared by AIDS $^{3}$. Recently, however, several typical cases were found in a survey of patients from Guinea Bissau and other countries of West Africa ${ }^{19-21}$. Unexpectedly, most of these patients did not have detectable titres of antibodies against HIV. But they were found to be infected by a retrovirus related to HIV by its ultrastructural and biological properties, such as cytopathogenicity and tropism for cells carrying the CD4(T4) antigen ${ }^{19}$. Antibodies raised against HIV could immunoprecipitate the $\mathrm{gag}$ and $\mathrm{pol}$ products of these isolates, which have molecular masses that are similar but not identical to these antigens of HIV; in contrast, the env products could not be immunoprecipitated, whereas previous HIV isolates showed wide cross-antigenicity of the envelope glycoprotein. Furthermore, the genome of this new retrovirus cross-hybridized only poorly in very low stringency conditions with HIV DNA probes ${ }^{19,22}$. We have therefore designated this West African AIDS virus as HIV type 2 (HIV-1 referring to the AIDS retrovirus previously identified in Central Africa, North America and Europe). More than 20 isolates have so far been made from patients with AIDS and related conditions, mainly originating from west Africa ${ }^{20,21}$, but also in some Europeans (L.M., unpublished), and epidemiological studies in progress indicate a seroprevalence of $1-2 \%$ in some populations of West Africa (F. Brun-Vézinet, personal communication).

HIV-2 appears to be closely related to the simian immunodeficiency viruses (SIV) a group of cytopathic retroviruses whose prototype, STLV-3 ${ }_{\text {mac }}$, was identified in captive rhesus monkeys (Macaca mulatta) with an AIDS-like disease ${ }^{23}$, and was later found to infect other primate species, either wild or in captivity $^{24-26}$. Genetic comparisons of SIV, HIV-1 and HIV-2 may help to elucidate the phylogeny of these viruses and the origins of the recent AIDS epidemics. As these retroviruses share most of their biological properties, the identification of conserved

\footnotetext{
* Present address: Laboratory of Molecular Microbiology, National Institute of Allergy and Infectious Diseases, Bethesda, Maryland, USA
}

† To whom correspondence should be addressed. sequences is important to localize the functional domains of the viral proteins and regulating elements, and design new diagnostic and therapeutic tools. We present here the complete nucleotide sequence of HIV-2, the comparison of its proteins with those of HIV-1, and preliminary studies on the regulation of HIV-2 expression.

\section{Nucleotide sequence and LTR analysis}

The sequence presented in Fig. 2 is derived from two $\lambda$ clones corresponding to integrated proviral DNA from the ROD isolate of HIV-2 (ref. 22), obtained in 1985 from an AIDS patient from Cape Verde Islands (offshore Senegal, refs 19, 20). The genome of HIV-2 is 9,671 nucleotides long (in its RNA form), whereas HIV-1 isolates are about 9,200 nucleotides long. This difference is partly explained by the respective sizes of the long terminal repeats (LTRs, see below).

The genetic organization of HIV-2 (shown in Fig. 1) is analogous to that of HIV-1, that is:

\section{5'LTR-gag-pol-central region-env-orf F-3'LTR.}

The 'central region', also identified in the ovine lentivirus visna ${ }^{27}$, contains five major open reading frames (ORFs), four being clearly related to the ORFs of HIV-1 that encode the Q (or sor), $\mathrm{R}$, tat and art (or trs) genes of HIV-1 (refs 15-18, 27-31). The fifth, which we designate ORF X, has no obvious counterpart in HIV-1. Alignments of the nucleotide sequences of HIV-1 and 2 show their distant homology (from $\sim 60 \%$ for the more conserved $\mathrm{gag}$ and $\mathrm{pol}$ genes, to $30-40 \%$ for the other viral genes and LTRs). To allow these alignments to be made many insertions and deletions must be introduced into the sequences. We do not find that these insertions are the small duplications that would be characteristic of the recent divergence of retroviral sequences, as was noted among isolates of HIV-1 (ref. 12).

The limits of the LTRs and of their internal U3, R and U5 elements, determined by sequence analysis and some complementary experiments, are shown in Fig. 2. Classically bounding the retroviral LTRs are short inverted repeats ( $5^{\prime}$ CTG-CAG $3^{\prime}$ ) located after a polypurine tract for the $3^{\prime} \mathrm{LTR}$, and before a sequence complementary to the $3^{\prime}$ end of a transfer RNA that is used as primer by the reverse transcriptase (here, as in HIV-1 and visna virus, a lysine tRNA, refs 15,27 ) for the 5' LTR. The R-U5 junction, corresponding to the $3^{\prime}$ end of the polyadenylated viral RNA, was previously localized by sequencing oligo(dT)-primed complementary DNA (cDNA) derived from the HIV- $2_{\text {ROD }}$ genome ${ }^{22}$. The length of U $5+R$, and hence the position of the U3-R junction corresponding to the $5^{\prime}$ cap site of the viral RNA were deduced from the size of a HIV-2 cDNA synthesized using the endogenous reverse transcriptase activity and the endogenous tRNA ${ }^{\text {lys }}$ primer (see Fig. 3). This 

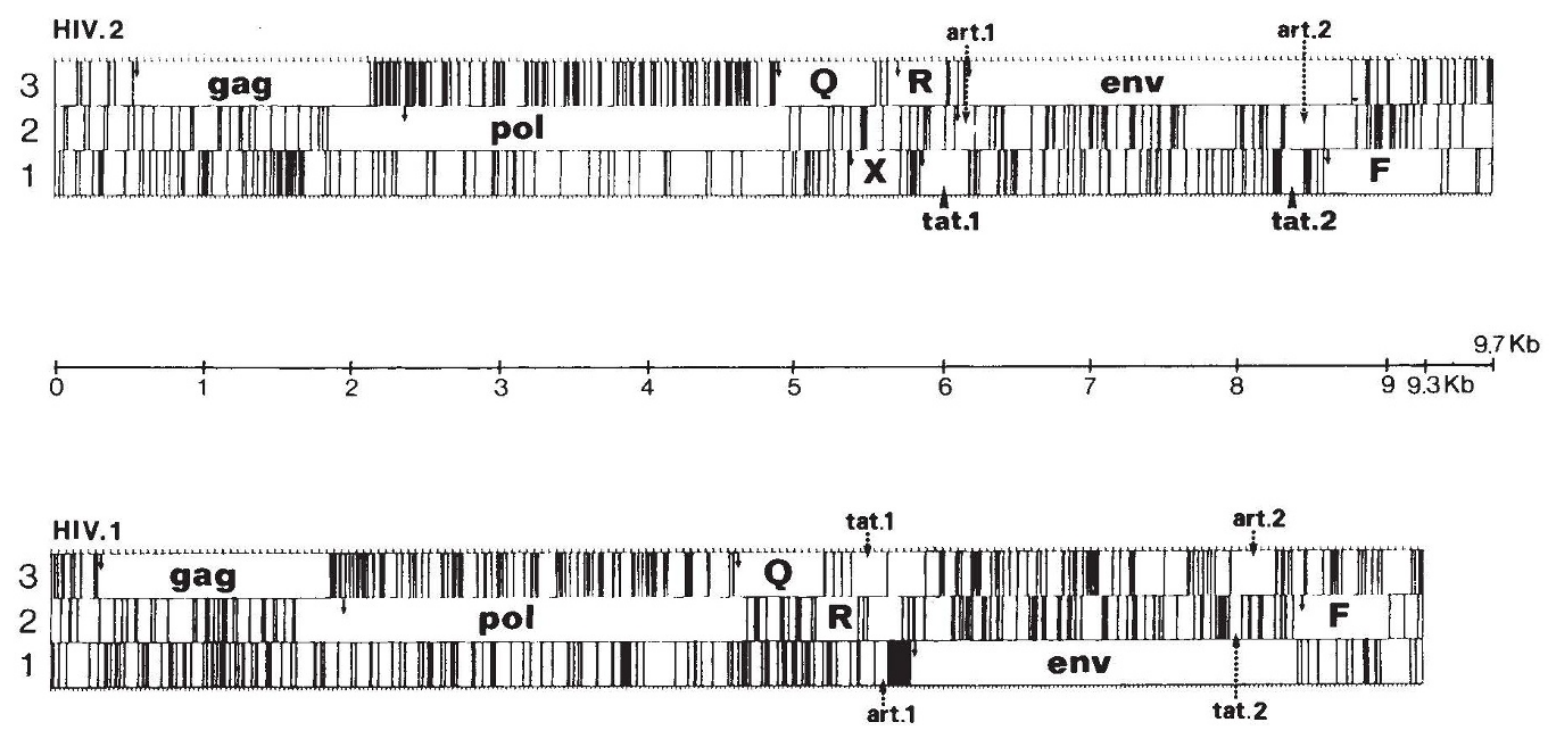

Fig. 1 Organization of the HIV-2 and HIV-1 genome (BRU isolate, ref. 15). Vertical bars represent the stop codons in the 3 reading frames. Arrows indicate the initiator AUG codons in viral genes or potential genes. Tat 1 and 2, art 1 and 2 are the open reading frames containing the coding exons of the tat and art genes.

'strong-stop cDNA' is $302+/-1$ nucleotides long (181 nucleotides in HIV-1, ref. 15). Thus, the U5 element is $125 \mathrm{bp}$ long, U3 is 556 bp and R 173 bp (respectively 82, 456 and $97 \mathrm{bp}$ in HIV-1). All the elements of the HIV-2 LTRs are larger than in HIV-1, and alignment by computer programs shows large insertions and very distant overall homology for the aligned regions ${ }^{22}$. However, the three Sp1 binding sites identified in HIV-1 (ref. 32 ), are also present in HIV-2 from nucleotide 9,419 to 9,448 with 17 out of 29 nucleotides homologous to this region of HIV-1. The core enhancers identified in HIV-1 (ref.33) are present in HIV-2 from nucleotide 9,389 to 9,416: the first is $50 \%$ homologous and the second $100 \%$ homologous to that in HIV-1 (Fig. 2).

The analysis of the virus-specific poly $(\mathrm{A})^{+}$RNA (not shown) from a cell line infected with and continuously producing HIV-2 revealed a pattern of transcription reminiscent of that observed in HIV-1-infected cells: RNA of over 9 kilobases (kb), corresponding to a full-length transcript, and three types of spliced messenger RNA of 5, 4.5 and $2 \mathrm{~kb}$, also observed in HIV-1 (refs $18,34)$.

\section{The gag and pol proteins and HIV phylogeny}

The gag precursor of HIV-2 has a calculated relative molecular mass of $57,100\left(M_{\mathrm{r}} 57.1 \mathrm{~K}\right)$, consistent with the p55 antigen ${ }^{20}$ seen by immunoprecipitation with patient sera, and is probably processed, by analogy with HIV-1, into the proteins designated p16, p26 and p12 (refs 19,20). By analogy with the p18 gag of HIV-1, p16 would be at the amino terminus of gag and precede $\mathrm{p} 26$, whose amino terminus has been sequenced (H. Marquardt, personal communication) and starts with the proline residue at position 951. The carboxy-terminal part of the gag precursor encodes a p12 that contains the cysteine-rich consensus of the retroviral nucleic-acid-binding proteins also found twice in the p13 ${ }^{\text {gag }}$ of HIV-1 (ref. 15). The HIV-2 pol ORF could encode the p64 and p36 antigens of HIV-2 (ref. 20) which by analogy correspond to the p68 and p34 (reverse transcriptase and endonuclease, respectively ${ }^{35}$ ) of HIV-1.

The gag and pol proteins of HIV-1 and 2 were expected to share large conserved domains, as these HIV-2 proteins can be precipitated by antibodies in sera from patients infected with HIV-1. However, we found that only $58 \%$ and $59.4 \%$ of the amino acids of gag and pol respectively are identical to the corresponding HIV-1 products (Table $1 a$ ), whereas the more distant isolates of HIV-1 (Zairian and US) show 90 to $95 \%$ amino-acid identity in these proteins (Table $1 b$ and ref. 12). Several insertions and deletions have to be introduced in the alignments (data not shown), whereas they are rare in the comparisons of gag and pol genes between HIV-1 isolates. The gag and pol proteins of HIV-2 are no closer to those of the Zairian isolates than to the prototype HIV-1 (BRU isolate) isolated in 1983 from a French patient ${ }^{6}$ probably infected in the USA. Overall, the difference in gag and pol between HIV-1 and HIV-2 is of the same order as that observed among the group of the human T-cell leukaemia viruses (HTLV-I and II) and bovine leukaemia virus (BLV). However, this latter group displays a higher conservation in the envelope, $70 \%$ amino-acid identity between HTLV-I and HTLV-II, versus about $42 \%$ between HIV-1 and HIV-2 (see below). Alignments of different retroviral pol proteins (Table $1 b$ ) confirm that the HIVs form a subgroup that is more related to the lentiviruses visna and equine infectious anaemia virus (EIAV) than to any other human or animal retrovirus.

\section{Homologous domains in env}

The envelope glycoproteins of retroviruses are translated from a subgenomic viral mRNA (here probably the transcript of $4.5 \mathrm{~kb}$ ). Addition of sugar residues ( $N$-linked glycosylation) gives rise to a high- $M_{\mathrm{r}}$ precursor which is processed by proteolytic cleavage. The length of the leader sequence of the HIV-2 glycoprotein cannot be precisely determined by alignment with that of HIV-1 (experimentally found to be 32 amino acids long ${ }^{36}$ ) because of a lack of sequence homology (Fig. 4). But the amino terminus of env contains a relatively hydrophobic stretch in the calculated hydropathy plot (not shown) that is probably the signal peptide. The potential cleavage site between the external envelope glycoprotein $(120 \mathrm{~K})$ and the transmembrane protein (previously thought to be the $36 \mathrm{~K}$ antigen ${ }^{19}$, and now putatively identified as a $40 \mathrm{~K}$ antigen ${ }^{20}$ ) is found at amino acid 505 (Fig. 4) immediately after the Lys-Glu-Lys-Arg sequence. This cleavage site aligns partly to one (Lys-Ala-Lys-Arg) of the two potential cleavage sites found in HIV-1 (the other being located after the Arg-Glu-Lys-Arg stretch). The calculated $M_{\mathrm{r}}$ of the extracellular glycoprotein (EGP) and of the transmembrane protein (TMP) of HIV-2 would be $57 \mathrm{~K}$ and $41.7 \mathrm{~K}$ respectively; the discrepancy 
$\rightarrow R$ GCCCCAG

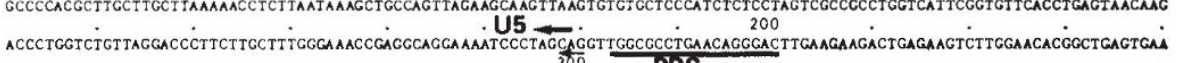

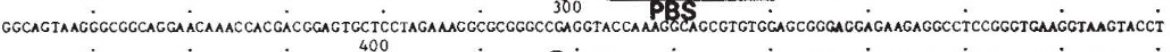

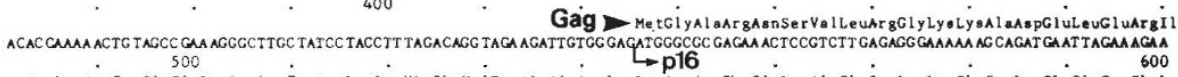

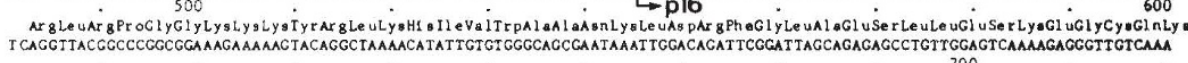

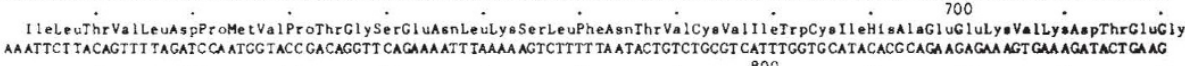

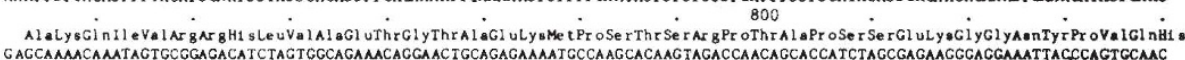
GACCAMMCAMATAOCOGAOCACTGG

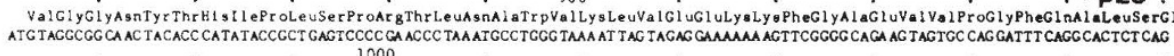

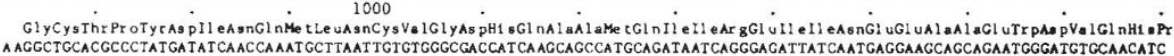

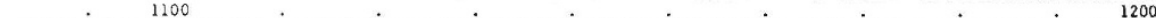

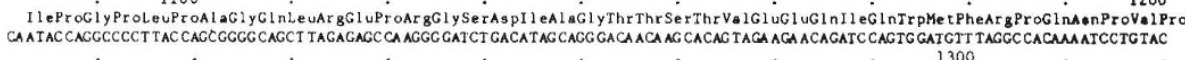

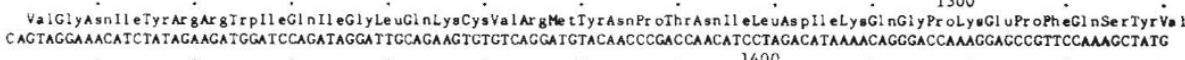

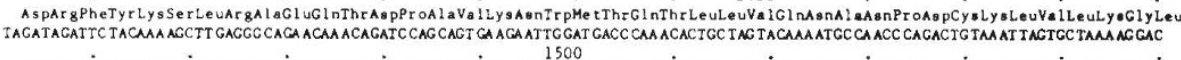

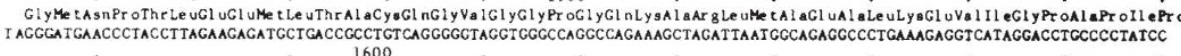

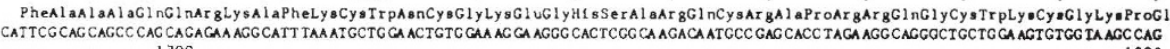

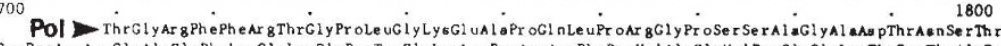

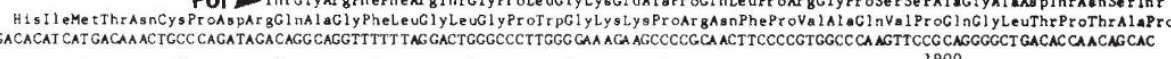

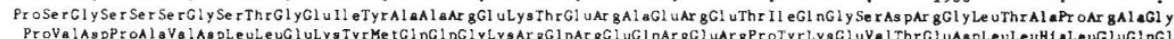

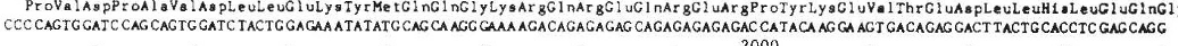

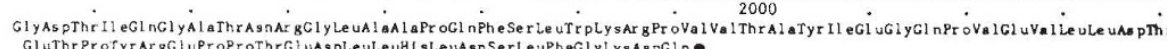

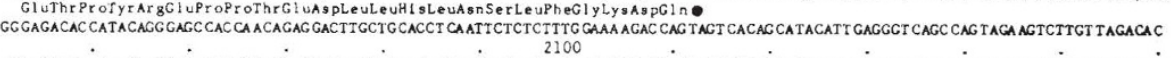

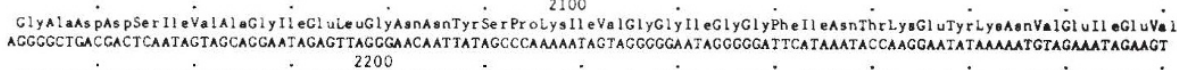

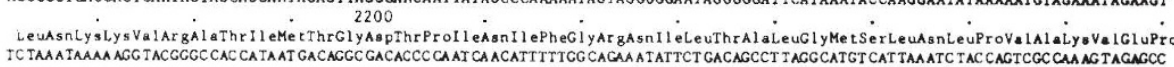

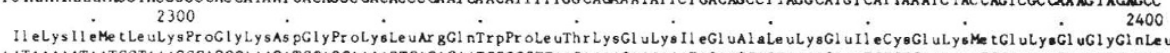
G AGAAAAATAGAAGCACTAAAAGAAATCTGTGAAAAATGGAMAAGMGGCCAGCT

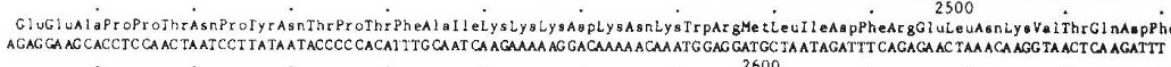

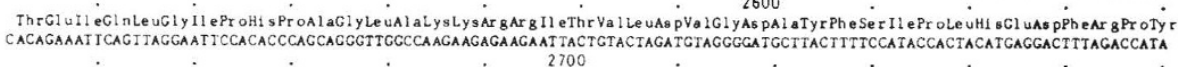

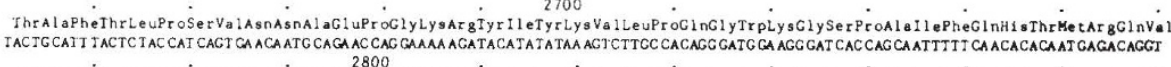

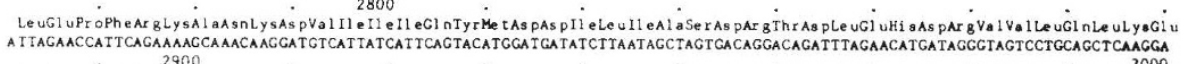

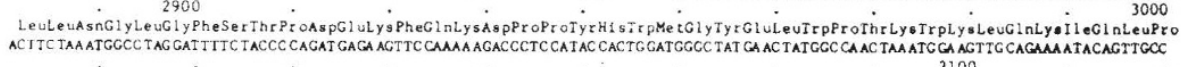

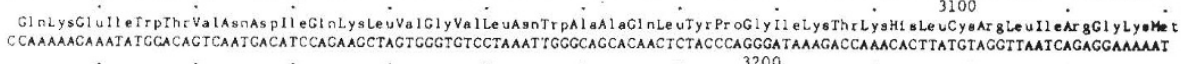

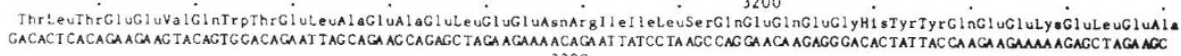

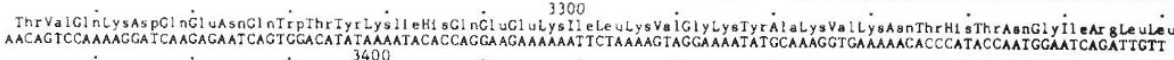

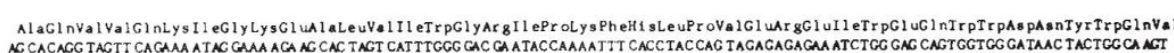
${ }_{3500}{ }_{0}$

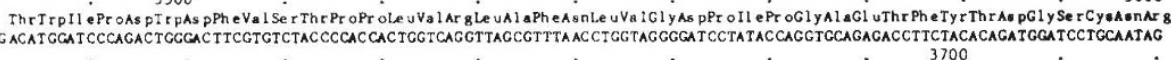

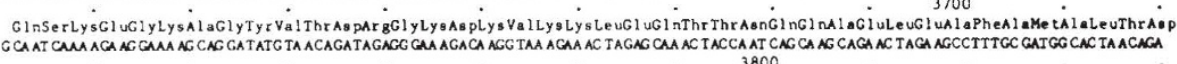

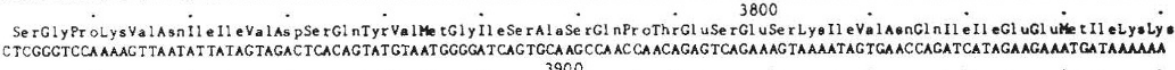

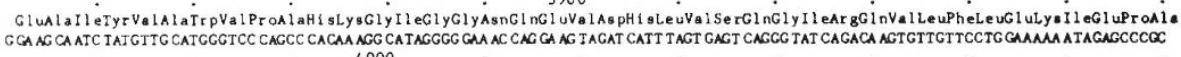

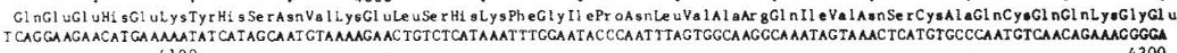

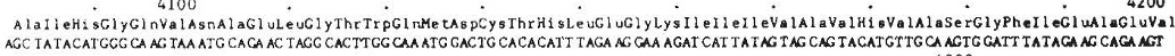

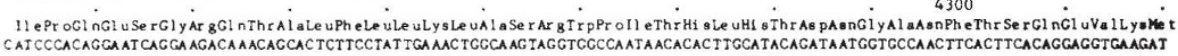

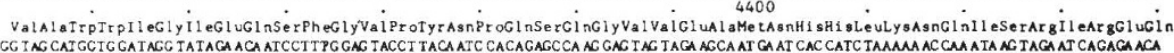

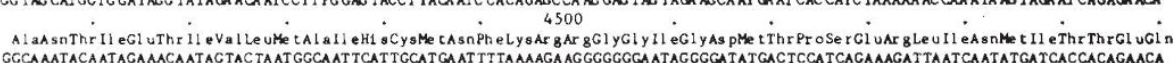

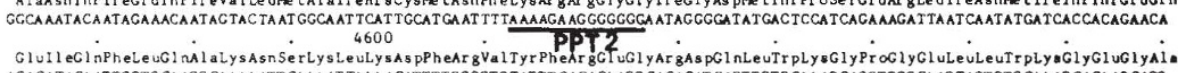

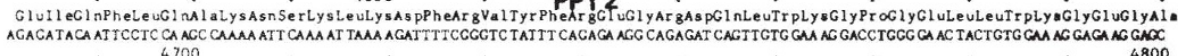

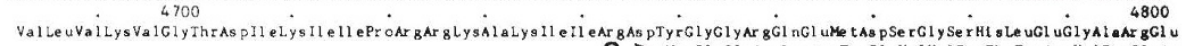

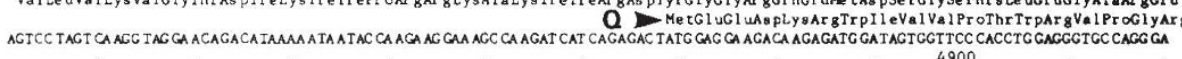

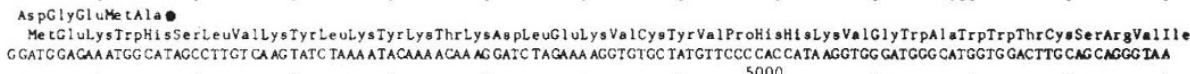

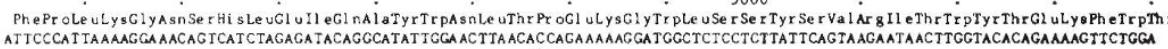
ATTCCCATTAAAGGAAACAGTCATCTAGAGATACAGGCATATTGGAACTTAACACCAGAAAAGGATGCCTCTCCTCTTATTCAGTAAGATAACTTGGACACAGAMAGTTCTGG

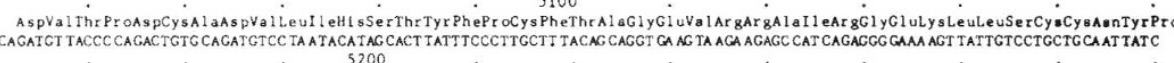

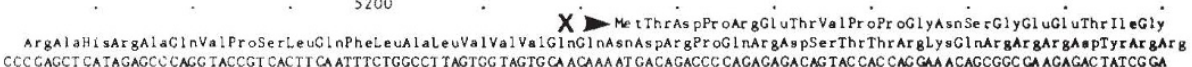

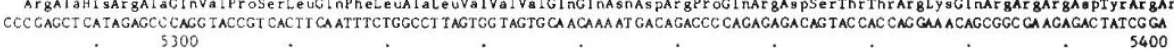

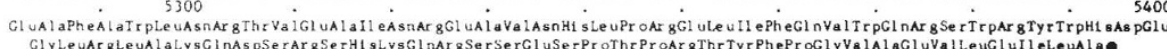

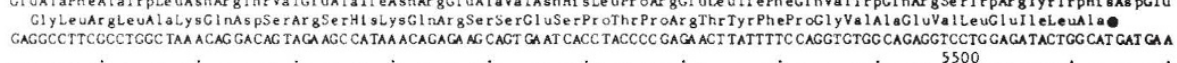

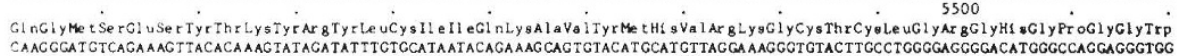

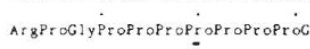
5600

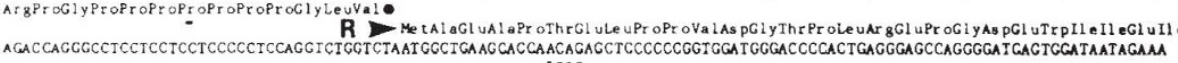

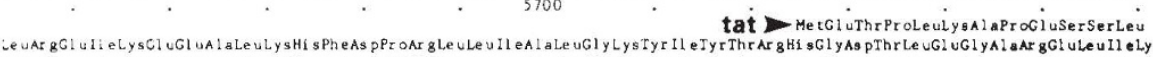

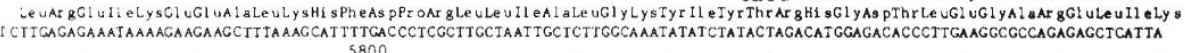

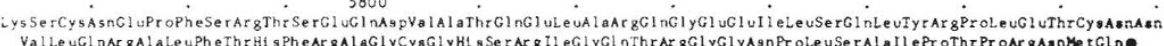

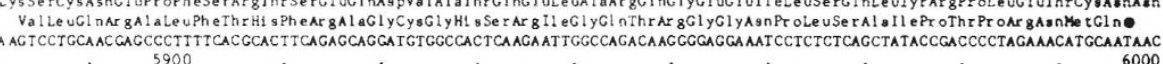

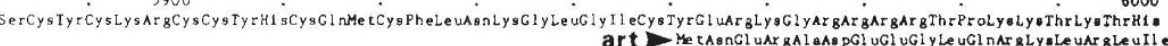

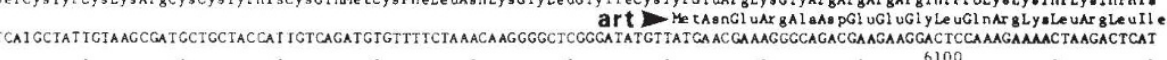

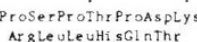

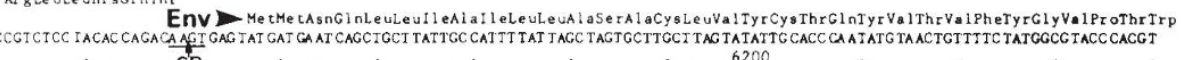

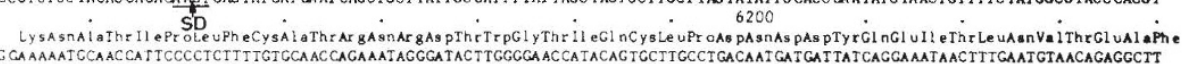

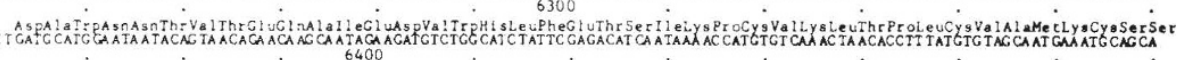




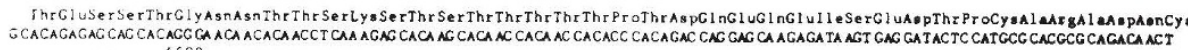

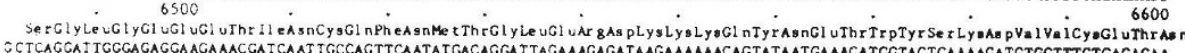

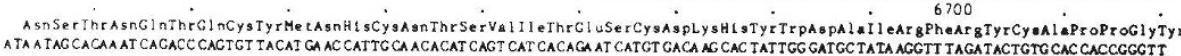

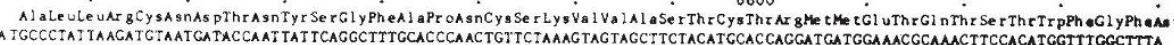

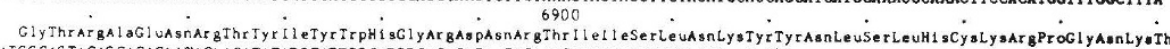

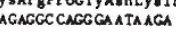

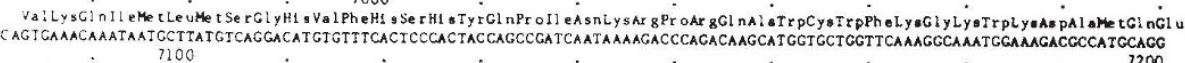

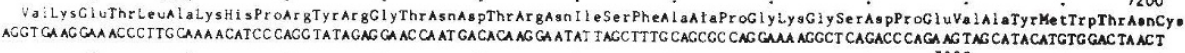

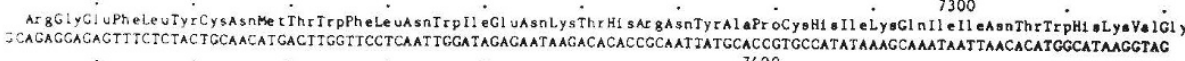

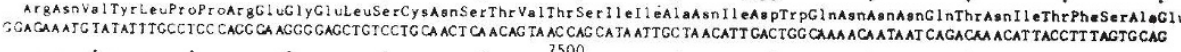

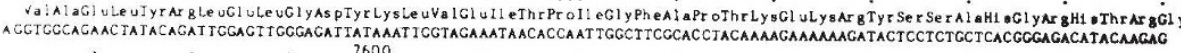

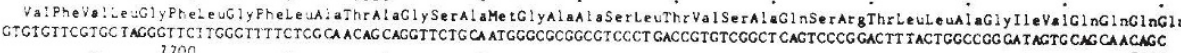

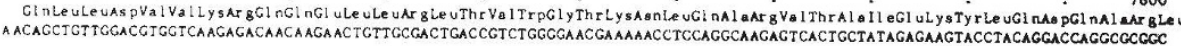

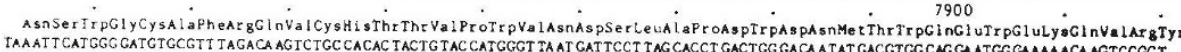
8000 . 800 .

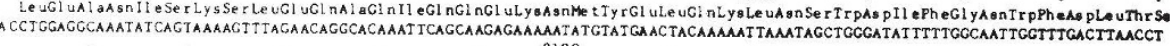

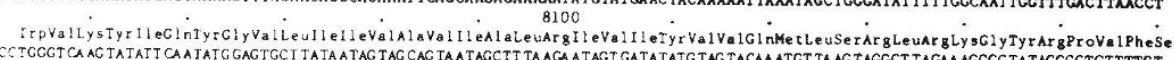

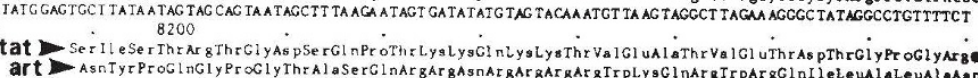

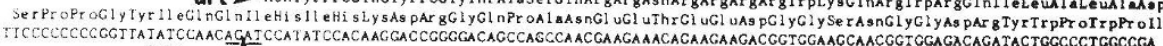
$8300 \frac{5}{5}$ A

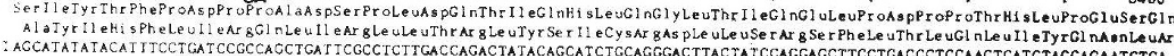

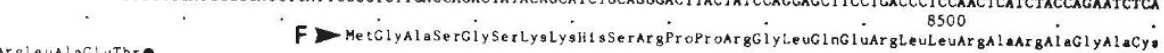

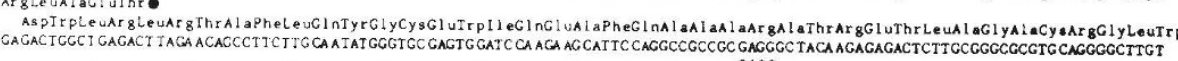

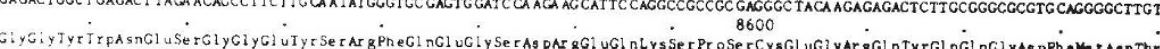

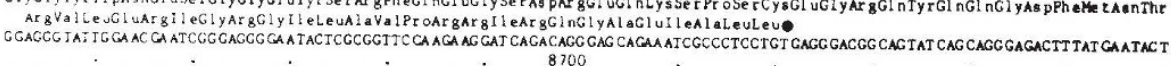

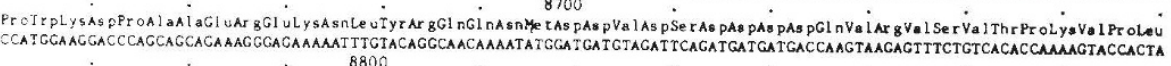

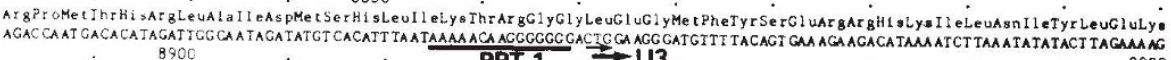

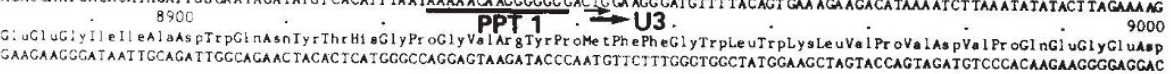

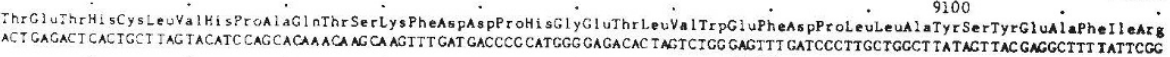

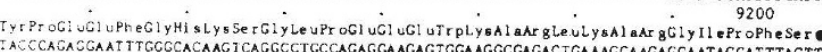

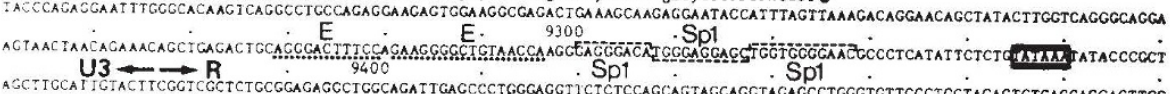

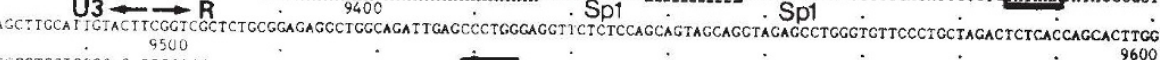

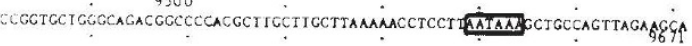

Fig. 2 Complete nucleotide sequence of the HIV-2 of the 9,671 nucleotide coding strand from the cap site to the polyadenylation site of the RNA is shown together with the deduced amino-acid sequence of the viral proteins. The 3-bp inverted repeats bounding the LTR are underlined with arrows. The primer binding site (PBS) complementary to the $3^{\prime}$ end of tRNA ${ }^{\text {lys }}$, and polypurine tract (PPT) are underlined. The repeat of the PPT in the pol gene is also underlined. The limits of the U3, $\mathrm{R}$ and U5 elements of the LTR are indicated by arrows. The promoter (TATAAA), the three potential $\mathrm{Sp} 1$ factor binding sites (indicated by dotted lines) and the two core enhancer equences (E, indicated by dashed lines) of U3 are shown; in R the polyadenylation signal AATAAA is underlined. The viral genes and potential genes are translated from the first AUG of the open reading frame (ORF), except pol which is translated from the beginning of the ORF. The end of translation is indicated by a filled circle. The amino terminus of the major core protein, the $\mathrm{p} 26^{\mathrm{gag}}$ was determined by protein sequencing. SD at position 6,140 and SA at position 8,307 indicate the probable splice donor and acceptor sites of the intron separating the two coding exons of the tat and art genes.

Methods. The sequence was determined by the M13 shotgun cloning and dideoxynucleotide chain terminator method ${ }^{57,58}$, as described ${ }^{27}$ starting from the inserts of the $\lambda$ phages ROD 27 and ROD 35 containing integrated proviral DNA 22 . ROD 27 corresponds to the $5^{\prime}$ part of the genome, to the EcoRI site at position 2,658, whereas. ROD 35 corresponds to the part of the genome $3^{\prime}$ to this site. An in-frame TAG stop codon was found in ROD 35 env gene. The sequencing of the corresponding region in an oligo(dT)-primed HIV-2 cDNA (clone E2, described in ref. 22) revealed that it was due to a C-to-T mutation at position 8,304. 


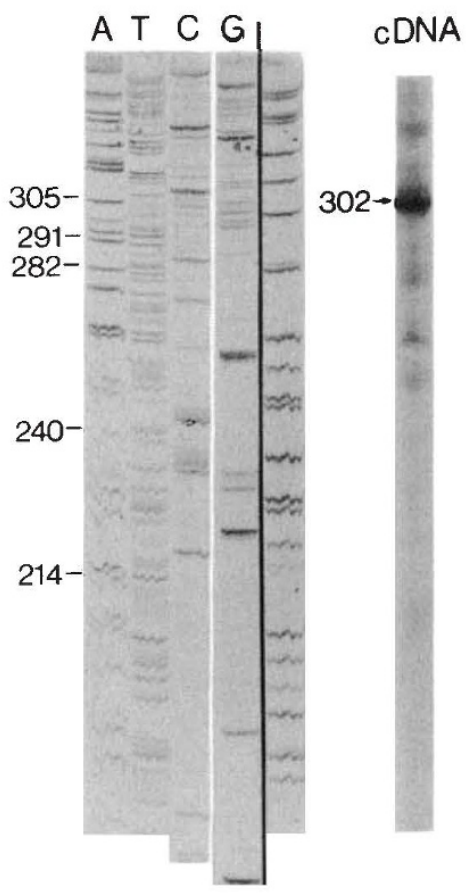

Fig. 3 HIV-2 strong-stop cDNA corresponding to the length of the R+ U5 elements of the HIV-2 LTR. The methods were previously described ${ }^{15}$. Briefly, virions were purified by ultracentrifugation and an endogenous CDNA reaction performed with radiolabelled nucleotides after mild disruption of viral envelope with Triton X-100. The tRNA ${ }^{\text {lys }}$ primer, complementary to the PBS site flanking the U5 element at the $5^{\prime}$ end of the genome, was then degraded by alkaline hydrolysis and the cDNA run on a denaturing $6 \%$ acrylamide-urea gel together with a sequence reaction for accurate estimation of the size of the products.

with the apparent $M_{\mathrm{r}}$ of the EGP is explained by glycosylation ( 30 sites in HIV-2, about half of which are conserved with respect to HIV-1).

Figure 4 shows an alignment of the envelopes of the two HIVs. The proteins are overall very distantly related $(41.7 \%$ identity in the entire envelope, $39.4 \%$ in the EGP, $44.8 \%$ in the TMP) compared to divergent isolates of HIV-1 (about $75-80 \%$ identity in the whole envelope, ref. 12). Many large insertions have to be introduced, particularly in alignment of the EGPs where only short, widely separated domains are conserved between HIV-1 and 2. These domains are clustered into the conserved regions of the EGP of HIV-1 (identified by comparison of different isolates ${ }^{12-14}$ ), and generally coincide with cysteine residues. Among the HIV-1 isolates, all the cysteine residues could be aligned in spite of the generally large genetic variation, especially in gp110. Almost all $(22 / 23)$ of the cysteine residues of HIV-1 can also be aligned with HIV-2, but the latter contains seven additional cysteine residues, often in the regions representing insertions relative to HIV-1. Thus, the folding of the HIV-2 EGP could be different from that of HIV-1, and some regions, therefore, might be exposed in a different manner.

\section{Other viral proteins}

The HIV-1 genome contains several other genes encoding proteins of small $M_{\mathrm{r}}(10$ to $27 \mathrm{~K})$, two of which (tat and art/trs) have an identified function: the positive regulation of viral expression $^{30-33}$. No role has yet been identified for the p23 encoded by ORF Q (or $s o r)^{37,38}$, nor for the p 27 encoded by ORF F (or 3' ORF) ${ }^{39}$. We also observed in the region between the $p o l$ and $e n v$ genes of HIV-1 (central region) another potential gene, which we designated $\mathrm{R}$ (ref. 12). All these elements are found in HIV-2, but the corresponding proteins are only distantly homologous (see Table $1 a$ ). In the F protein, most of the difference between HIV-1 and 2 is due to a large insertion in the amino terminus of HIV-2. The second half of the protein, encoded by the U3 element of the LTR, shows better conservation (data not shown).

Based upon sequence homologies with HIV-1, the tat and art genes of HIV-2 are probably organized as split genes transcribed into $\sim 2 \mathrm{~kb}$ mRNA made of three exons ${ }^{18,28-31}$ : the $5^{\prime}$ leader, a first coding exon located in the central region and probably ending at a possible splice donor found at position 6,140 (CAAGT, Fig. 2), and a last exon probably starting at the splice acceptor at position 8,307 in HIV-2 (CAGATC). The tat protein of HIV-2 would be longer than that of HIV-1 (130 versus 86 amino acids), having two large insertions in the amino terminus and in the second coding exon (Fig. 4). The main domain of homology of the tat proteins corresponds to a region very rich in cysteine residues whose structure is reminiscent of that of the 'cysteine fingers' of some transcription-regulating elements that interact with nucleic acids, such as the TFIIIA factor ${ }^{40}$. This region is followed by an Arg-Lys-rich stretch that could also interact with DNA or RNA. No significant homology is seen in the second coding exon, which has been shown to be dispensable to the function of the protein ${ }^{28,29}$. The art-encoded protein is shorter in HIV-2 than it is in HIV-1 (100 versus 116 amino acids), and most of its length is encoded by the last exon. The most homologous part is located in a stretch of basic residues that may be able to interact with nucleic acids.

\section{Cross-transactivation of HIV-1 and HIV-2}

The trans-activator gene (tat) has been shown to be indispensable for the replication and cytopathicity of HIV-1 (ref.41).

Table 1 Quantification of the homologies among retroviral proteins

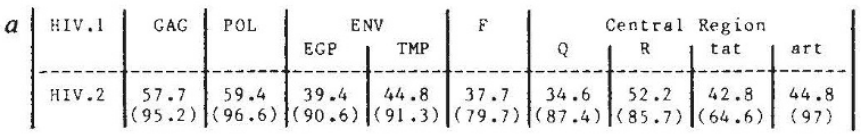

\begin{tabular}{|c|c|c|c|c|}
\hline$b$ & HIV-2 & HIV-1 & HTLV-I & VISNA \\
\hline HIV-1 & $\begin{array}{c}59.1 \\
(96.4)\end{array}$ & - & ND & ND \\
\hline LAV-Eli & $\begin{array}{c}61.6 \\
(96.1)\end{array}$ & $\begin{array}{c}94 \\
(98.7)\end{array}$ & ND & ND \\
\hline LAV-Mal & $\begin{array}{c}59 \\
(95.2)\end{array}$ & $\begin{array}{c}92 \\
(98.7)\end{array}$ & ND & ND \\
\hline EIAV & $\begin{array}{l}43.8 \\
(92)\end{array}$ & $\begin{array}{c}41.9 \\
(91.5)\end{array}$ & ND & $\begin{array}{c}46.7 \\
(90.8)\end{array}$ \\
\hline VISNA & $\begin{array}{c}43.7 \\
(88.7)\end{array}$ & $\begin{array}{l}42.2 \\
(94)\end{array}$ & ND & - \\
\hline HTLV-I & $\begin{array}{c}34.8 \\
(70.5)\end{array}$ & $\begin{array}{c}33.3 \\
(70.3)\end{array}$ & - & ND \\
\hline HTLV-II & ND & ND & $\begin{array}{c}62.8 \\
(99.5)\end{array}$ & ND \\
\hline BLV & ND & ND & $\begin{array}{c}49.5 \\
(93.2)\end{array}$ & ND \\
\hline RSV & $\begin{array}{c}35.9 \\
(72.3)\end{array}$ & $\begin{array}{c}34.5 \\
(76.2)\end{array}$ & $\begin{array}{c}38.2 \\
(86.4)\end{array}$ & ND \\
\hline
\end{tabular}

The reference protein of each alignment is that listed at the top of the column. Proteins were aligned using the NUCALN program ${ }^{61}$ with following parameters: K-tuple 1 , window 20 , gap penalty 1 . Two results are indicated in each case: the amino-acid identity $(\%)$ in the aligned domains (that is, excluding the regions of insertion/deletion), and between parentheses the percentage of the length of reference protein that could be aligned. $a$, Homologies between HIV-1 and HIV-2 proteins. For env, the calculation was done for the external glycoprotein (EGP, including the signal peptide, whose length is not exactly known in HIV-2), and the transmembrane protein (TMP). $b$, Comparison of the pol-encoded proteins of different retroviruses. LAV.Mal and LAV.Eli are Zairian isolates of HIV-1 (ref. 12); EIAV: equine infectious anaemia virus (sequence communicated by $\mathrm{Dr} \mathrm{S}$. Aaronson), and visna virus ${ }^{27}$ are animal lentiviruses; HTLV-I, HTLV-II, BLV ${ }^{62-64}$, related leukaemogenic retroviruses; RSV) Rous sarcoma virus ${ }^{65}$. ND, not determined. 


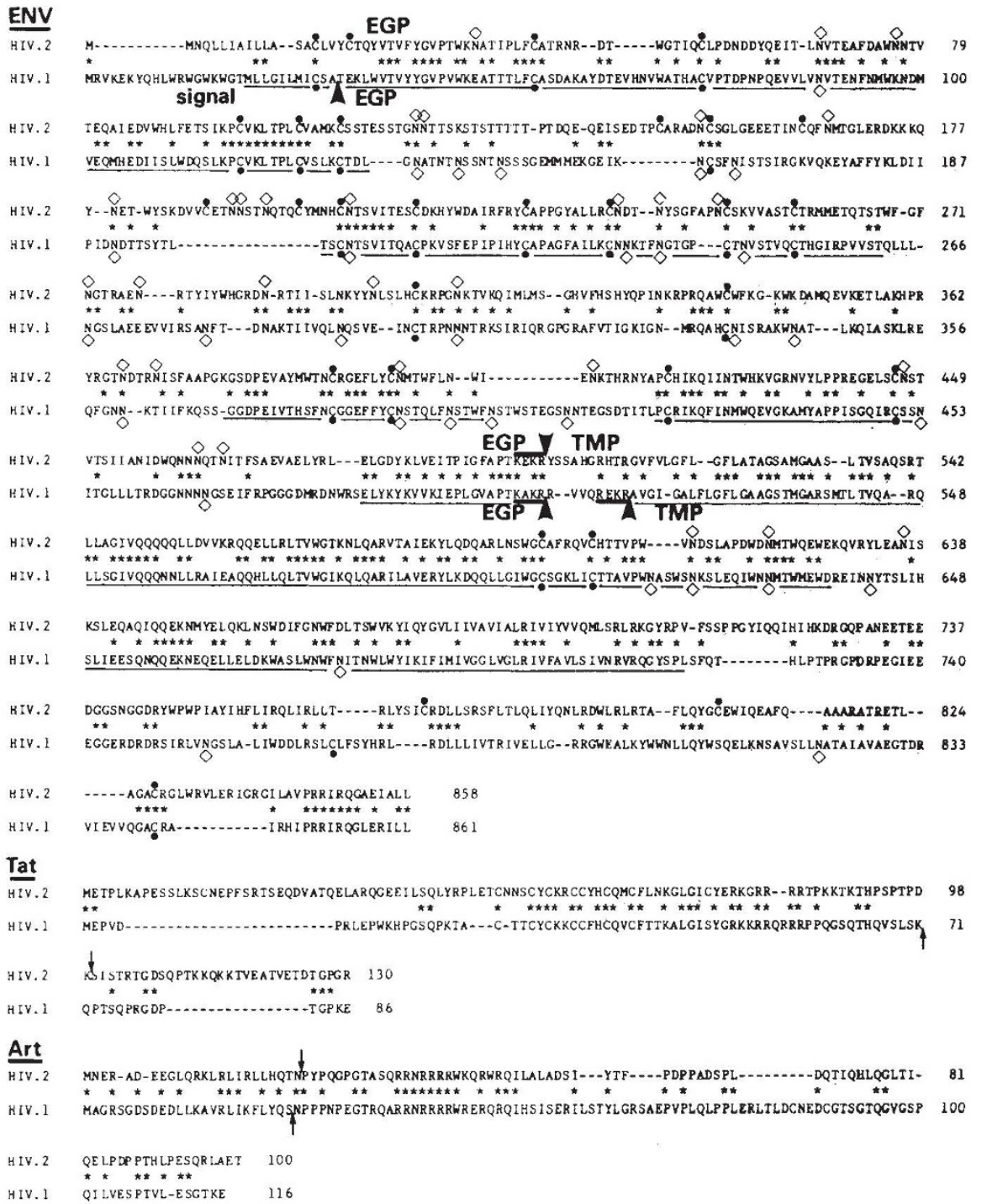

To examine whether transactivation (a property also shared with the ovine visna lentivirus but not with the related caprine arthritis and encephalitis virus ${ }^{42}$ ) exists in HIV-2, we constructed a plasmid, called pHIV2-CAT, containing the bacterial chloramphenicol acetyltransferase (CAT) gene under the control of the U3-R region of HIV-2 (225 bp of U3 and $175 \mathrm{bp}$ of R). To test the transactivation of HIV-2, cells were either infected with HIV-2 or mock-infected, and five days later transfected with either pSVCAT (which contains the CAT gene under control of the SV40 early promoter ${ }^{43}$ ) or pHIV2-CAT. At the time of transfection, the cells were not producing virus. Nonetheless, we observed a substantial increase in the amount of CAT expression in extracts of HIV-2-infected versus mock-infected cells that had been transfected with pHIV2-CAT (Fig. $5 a$ ). The expression of the SV40 early promoter was not affected by HIV-2 infection.

To determine whether the tat gene of HIV-1 could transactivate the LTR of HIV-2 and vice versa, we cotransfected SW480 cells $^{44}$ with subgenomic fragments of HIV-1 or HIV-2 and pHIV2-CAT or a plasmid called pHIV1-CAT, which contains U3-R of HIV-1 (the entire U3 and $70 \mathrm{bp}$ of R) directing transcription of the CAT gene. The plasmid pLET (a gift from Dr S. Wain-Hobson) contains the region of the HIV-1 shown by others to encode the HIV-1 tat gene ${ }^{28,29}$. The plasmid pME214, on the other hand, contains HIV-2 sequences between nucleotides 5,786 and 8,571 (Fig. 2), and in particular contains the open reading frames of HIV-2 that share homology with the tat gene of HIV-1. In both of these plasmids transcription is driven by the LTR of the respective virus, and the first AUG of the transcript is the first AUG of the putative tat gene. It should be noted that both these plasmids also contain the coding potential for the art gene.

Although the SV40 early promoter was not affected by either the HIV-1 tat nor the HIV-2 tat genes, both HIV-1 and HIV-2 LTRs were substantially activated by the HIV-1 tat gene (Fig. $5 b$ ). This is perhaps surprising in view of the difference in size of the $\mathrm{R}$ region of HIV-1 (where the transactivator responsive region (TAR) resides ${ }^{45}$ ) and HIV-2. However 35 of the 58 bases present in the first stem-and-loop secondary structure of the TAR region of HIV-1 are conserved, and an analogous stem-and-loop structure with the first 77 bases of $R$ can be drawn for HIV-2 (ref. 33).

The HIV-2 LTR is transactivated over 100 -fold by pME214 (Fig. $5 b$ ). On the other hand, the HIV-1 LTR is not as well transactivated by this plasmid $(\sim 5-20$ fold, Fig. 5 and other data not shown). Similar results were obtained after transfection of HeLa and HUT 78 cells (data not shown). These experiments indicate that pME214 encodes a functional tat gene. In addition, they indicate that the specificity of the HIV-2 tat is somewhat different from that of the HIV-1 tat. It will be important to determine whether this observation is isolate-specific.

\section{Origin of human immunodeficiency viruses}

We have presented here the complete nucleotide sequence of the retrovirus associated with AIDS in West Africa, HIV-2, and tentatively identified the viral proteins either detected in 


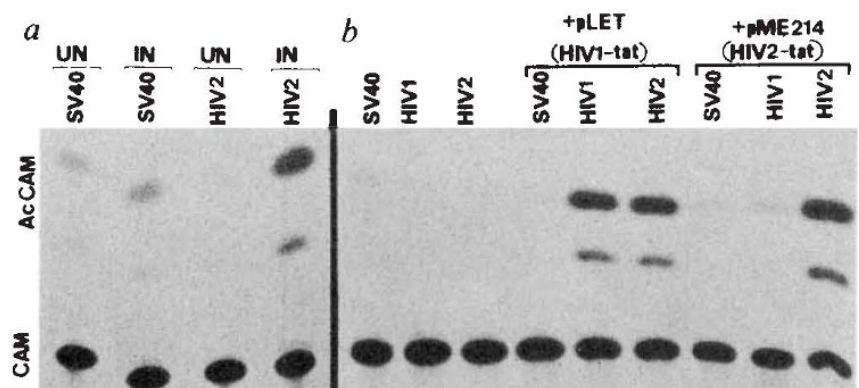

Fig. 5 Transactivation of HIV-2. Chloramphenicol acetyltransferase (CAT) assays were done as described ${ }^{59}$. The unreacted chloramphenicol is marked 'CAM', and the acetylated products are marked 'AcCAM'. All reactions were $1 \mathrm{~h}$ with $10 \%$ of the cellular extract made $40 \mathrm{~h}$ after transfection. The origin of the promoter linked to the CAT gene is indicated above each lane. SV40 indicates the SV40 early promoter, HIV-2 indicates the partial U3 and the entire R sequences of HIV-2 (ROD isolate), and HIV-1 indicates the entire $\mathrm{U} 3$ and $70 \mathrm{bp}$ of R of HIV-1 (BRU isolate). $a$, HUT 78 cells were either mock-infected (UN, uninfected) or infected (IN) with HIV-2. Five days post-infection, $3 \times 10^{6}$ cells were transfected with $3 \mu \mathrm{g}$ of plasmid in $0.5 \mathrm{ml}$ of Tris-saline without divalent cations for $45 \mathrm{~min}$ at $37^{\circ} \mathrm{C}$ with $250 \mu \mathrm{g} \mathrm{ml}^{-1} \mathrm{DEAE}$ Dextran. $b, 4 \times 10^{5} \mathrm{SW} 480$ cells were cotransfected by the $\mathrm{CaCl}_{2}$ technique ${ }^{60}$ with $3 \mu \mathrm{g}$ of promoter-CAT plasmid and $3 \mu \mathrm{g}$ of the indicated plasmid. Salmon sperm DNA was added such that each transfection was $20 \mu \mathrm{g} \mathrm{ml}^{-1}$ DNA. This experiment was repeated three times with similar results.

immunoprecipitations with patients' sera, or homologous to proteins previously identified in HIV-1. The two viruses share a similar genomic organization, indicating a common evolutionary origin, but differ significantly in terms of nucleotide and amino-acid sequence: the more-conserved gag and pol genes respectively display only 56 and $60 \%$ nucleotide sequence homology and both less than $60 \%$ amino-acid identity. The calculation of the nucleotide sequence homology for the other genes gives even lower values, making HIV-1 and $242 \%$ homologous overall. This confirms that these two viruses are distinct elements of the HIV family, and cannot be considered as strains of the same virus, according to the recommendations of the international taxonomy committee ${ }^{46}$.

It was previously established that HIV-2 is more related to the simian immunodeficiency viruses (SIV) than it is to HIV-1. The gag, pol and env proteins of SIV and HIV-2 are antigenically cross-reactive, whereas their cross-reactivity to HIV-1 is restricted to some gag and pol antigens. The amino-terminal aminoacid sequence of the major core protein (corresponding to the p2 $5^{\text {gag }}$ of HIV-1 and p26 $6^{\text {gag }}$ of HIV-2) has been determined in one isolate of SIV obtained from macaques with an AIDS-like disease (MnIV, ref. 26). Out of the 23 amino acids sequenced 21 match with the amino terminus of $\mathrm{p} 26^{\mathrm{gag}}$ of HIV-2, whereas 13 (with one deletion) match to the p25 $5^{\text {gag }}$ of HIV-1. Furthermore, whereas HIV-2 can infect, at least transiently, primate species which are evolutionarily more distantly related to humans (at least baboons and macaques), HIV-1 infects only humans and chimpanzees (R. Desrosiers and P. Fultz, personal communications). In fact, it is not possible from current data to know whether SIV can be classified as distinct from HIV-2 or if they only differ as independent isolates of the same virus.

The almost simultaneous emergence of two foci of AIDS in distinct areas of the African continent is unlikely to be due to the recent emergence of two novel human pathogens, for example by simultaneous trans-species infection by animal retrovirus, or by the mutation of pre-existing non-pathogenic human retroviruses. Indeed, HIV-1 and HIV-2 are obviously retroviruses with a common origin, but they are highly divergent, and it is more likely that their time of divergence is earlier than the beginning of the current epidemics. Therefore a common ancestor, with similar properties and pathogenic potential, prob- ably existed a long time ago in a human population, and the emergence of the AIDS epidemics is more likely the result of simultaneous modifications of epidemiological parameters in West and Central Africa, such as uncontrolled urbanization, leading to the infection of larger populations.

A question to be addressed is why the HIVs were only recently detected if they existed for a long period. This may be due to the fact that the pathogenicity of an HIV-type retrovirus cannot be revealed until it has spread to a significant portion of the population. First, in areas of Africa with poor medical facilities, where other infections, such as malaria, represent primary causes of morbidity, isolated cases of AIDS could have been an undetectable clinical event. Then, the incubation time can vary considerably, and it cannot still be ruled out that a large fraction of individuals infected by a HIV will remain healthy carriers. In Kenya, HIV-1 seropositivity was first reported in a high fraction of subjects at risk of AIDS (female prostitutes) who were apparently healthy; later, the virus diffused to a larger part of the population, and cases of AIDS were observed ${ }^{47}$. A similar situation could explain the apparent lack of pathogenicity of the retrovirus designated HTLV-IV, but indistinguishable from HIV-2 and SIV by the antigenicity of its proteins ${ }^{19,48,49}$. The presence of HTLV-IV was identified only in apparently healthy individuals in West Africa, an area where we have observed several typical AIDS cases caused by HIV-2. It is possible that the apparent non-pathogenicity of HTLV-4 is due to a recent epidemic diffusion of HIV-2/HTLV-IV in the West Africa, where AIDS cases still represent a minor fraction of the infected and seropositive individuals, whereas HIV-1 has diffused in major cities of central Africa or the USA some time before.

\section{Implications for vaccines and diagnostics}

The risk that HIV-2-infected blood samples may not be detected by standard screens, currently based on the detection of antiHIV-1 antibodies, makes it important that a way of diagnosing HIV-2 infection is found. As the envelope, and especially its transmembrane part, represents the primary target of the host antibody response to the HIV infection (see ref. 1), antigens from the envelope of HIV-2 will significantly improve the spectrum of the screening tests, allowing the detection of samples infected by HIV-2, and perhaps by other as yet uncharacterized members of the HIV family.

As it shares most of the structural characteristics and biological properties of HIV-1, but displays significant genetic divergence, HIV-2 is a powerful tool in the study of the molecular biology of this group of retroviruses. Among the crucial biological properties common to both HIVs are tropism for CD4positive cells, and mechanisms of positive regulation of viral expression encoded by viral transactivating factors. We observed that the tat of HIV-1 activates the transactivation responsive (TAR) sequences as efficiently in both types of HIV, whereas the tat gene of HIV-2 is more efficient on the TAR elements of HIV-2. The tat proteins of HIV-1 and 2 have only short homologous sequences, and this will ease the dissection of their function by mutagenesis or using chemically synthesized peptides.

HIV-1 and probably HIV-2 recognize the CD4 surface molecule as a receptor on helper/inducer $\mathrm{T}$ lymphocytes and perhaps on other cells expressing the CD4 protein ${ }^{50-53}$. In HIV-1, this interaction is mediated by the external envelope glycoprotein (EGP; ref. 52), and an important problem is which of the domain(s) of this protein are involved in that interaction. Indeed, blocking this step of the virus life cycle, either by antibodies or drugs, could be an efficient means for preventing infection or blocking its spread. As the receptor is a constant cellular protein, we can postulate that the binding domain of the envelope is conserved among the CD4-tropic HIVs. The conserved domains of the EGP of HIV-1 and 2 are not numerous, and therefore it becomes possible to demonstrate their possible role in the virus-receptor interaction using a relatively limited 
set of site-directed mutations. Given the absence of antigenic cross-reactivity of the envelopes of the two HIVs, this CD4binding domain is probably not, or only poorly, immunogenicperhaps because of masking by glycosylation, poor exposure on the virion surface, or mimicking of 'self' antigens. Nevertheless, its presentation to the immune system out of context of the virion, that is, as a peptide, might induce a neutralizing antibody response that is not attained, or attained with only a low efficiency, with the complete native envelope from virions or expression systems ${ }^{54-56}$.

\section{Conclusion}

The comparative analysis of HIV-1 and 2 reveals major genetic differences between retroviruses that share many of their biological properties. They both cause AIDS, are cytopathic in vitro,

Received 4 March; accepted 19 March 1987.

1. Weiss, R. A. in RNA Tumor Viruses (eds Weiss, R., Teich, N., Varmus, H. \& Coffin, J.) Vol. II, 405-485 (Cold Spring Harbor Laboratory, New York, 1985).

2. Curran, J. W. et al. Science 229, 1352-1357 (1985).

3. Quinn, T.-C., Mann, J. M., Curran, J. W. \& Piot, P. Science 234, $955-963$ (1986).

4. Piot, P. et al Lancet ii, 65-69 (1984).

5. Clumeck, N. et al. N. Engl. J. Med. 310, 492-497 (1984).

6. Barré-Sinoussi, F. et al. Science 220, 868-870 (1983).

. Popovic, M., Sarngadharan, M. G., Read, E. \& Gallo, R. C. Science 224, 497-500 (1984).

8. Levy, J. A. et al. Science 225, 840-842 (1984).

9. Brun-Vézinet, F. et al. Science 226, 453-456 (1984)

10. Ellrodt, A. et al. Lancet i, 1383-1385 (1984).

11. Bailey, A. C. et al. Lancet i, 359-361 (1985)

12. Alizon, M., Wain-Hobson, S., Montagnier, L. \& Sonigo, P. Cell 46, 63-74 (1986).

13. Willey, R. L. et al. Proc. natn. Acad. Sci. U.S.A. 83, 5038-5042 (1986)

14. Starcich, B. R. et al. Cell 45, 637-648 (1986).

15. Wain-Hobson, S., Sonigo, P., Danos, O., Cole, S. \& Alizon, M. Cell 40, 9-17 (1985).

16. Sanchez-Pescador, R. et al. Science 227, 484-492 (1985).

17. Ratner, L. et al. Nature $313,227-284$ (1985)

18. Muesing, M. A. et al. Nature 313, 450-458 (1985).

19. Clavel, F. et al. Science 233, 343-346 (1986).

20. Brun-Vézinet, F. et al. Lancet i, 128-132 (1987)

21. Clavel, F. et al. N. Engl. J. Med. (in the press)

22. Clavel, F. et al. Nature 324, 691-695 (1986).

23. Daniel, M. D. et al. Science 228, 1199-1204 (1985).

24. Kanki, P. J., Alroy, J. \& Essex, M. Science 230, 951-954 (1985).

25. Benveniste, R. E. et al. $J$. Virol. $60,483-490(1986)$

26. Murphey-Corb, M. et al. Nature 321, 435-437 (1986)

27. Sonigo, P. et al. Cell 42, 369-382 (1985)

28. Arya, S. K., Guo, C., Josephs, S. F. \& Wong-Staal, F. Science 229, 69-73 (1985).

29. Sodroski, J., Patarca, R., Rosen, C., Wong-Staal, F. \& Haseltine, W. Science 229, 74-77 (1985)

30. Sodroski, J. et al. Nature 321, 412-417 (1986).

31. Feinberg, M. B., Jarret, R. F., Aldovini, A., Gallo, R. C. \& Wong-Staal, F. Cell 46, 807-817 (1986)

32. Jones, K., Kadonaga, J., Luciw, P. \& Tjian, R. Science 232, $755-759$ (1986). have a tropism for CD4-bearing cells and have elements transactivating the expression of viral genes acting at the LTR level. The evolutionary potential of these viruses is therefore striking, and we must ask whether other HIVs can emerge as long as a favourable epidemiological situation is provided. We must take advantage of the precise delineation of the conserved structures to understand their molecular biology and develop new therapeutic tools, especially immunoprophylactics.

We thank Drs S. Aaronson, F. Brun-Vézinet, R. Desrosiers, P. Fultz, H. Marquardt and M. Muesing for exchange of scientific information; Drs S. Wain-Hobson, M. L. Michel and A. Louise for providing plasmids; Bernard Caudron for help in preparation of the computer printouts; Dr. Lautaro Perez for discussion and encouragement. M.E. is a fellow of the Leukemia Society of America, Inc.

33. Muesing, M. Smith,

34. Rabson, A. B. et al. Science 229, 1388-1390 (1985)

35. Lightfoot, M. et al, J. Virol. 60, 771-775 (1986)

36. Allan, J. S. et al Science 228, 1091-1094 (1985).

38. Kan, N. C et al Science 231, 1553-1555 (1986).

39. Allan, J. S. et al. Science 230, 810-813 (1985).

40. Miller, J., Mac Laehlan, A. \& Klug, A. EMBO J. 4, 1609-1614 (1985)

41. Dayton, A., Sodroski, J. G., Rosen, C. A., Goh, W. C. \& Haseltine, W. A. Cell 44, 941-947 (1986)

42. Hess, J. L., Pyper, J. M. \& Clements, J. E. J. Virol. 60, 385-393 (1986)

43. Gorman, C. M., Merlino, G. T., Willingham, M. C., Pastan, I. \& Howard, B. H. Proc. natn. Acad. Sci. U.S.A. 79, 6777-6781 (1982)

44. Adachi, A. et al. J. Virol. 59, 284-291 (1986)

45. Rosen, C. A., Sodroski, J. G. \& Haseltine, W. A. Cell 41, 813-823 (1985).

46. Coffin, J. M. et al. Nature 321, 10 (1986).

47. Kreiss, J. K. et al. N. Engl. J. Med, 314, 414-418 (1986)

48. Kanki, P. J. et al. Science 232, 238-243 (1986)

49. Barin, F. et al. Lancet ii, 1387-1389 (1985)

50. Dalgleish, A. G. et al. Nature 312, $763-767$ (1984)

51. Klatzmann, D. et al. Nature 312, 767-768 (1984).

52. McDougal, J. S. et al. Science 231, 382-385 (1985).

53. Maddon, P. J. et al. Cell 47, 333-348 (1986).

54. Weiss, R. A. et al. Nature 316,69-72 (1985)

55. Clavel, F., Klatzman, D. \& Montagnier, L. Lancet i, 879-880 (1985)

56. Lasky, L. A. et al. Science 223, 209-212 (1985).

57. Messing, J. \& Viera, J. Gene 19, 269-276 (1982).

58. Sanger, F., Nicklen, S. \& Coulson, A. R. Proc, natn. Acad. Sci. U.S.A. 74, 5463-5467 (1977)

59. Gorman, C. M Moffat, L. F. \& Howard B. H Molec Cell Biol 2, 1044-1051 (1982)

60. Graham, F. L. \& Van der Eb, A. J. Virology 52, 456-467 (1973).

61. Wilbur, W. J. \& L.ipman, D. J. Proc. natn. Acad. Sci. U.S.A. 80, 726-730 (1983),

62. Seiki, M., Hattori, S., Hirayama, Y. \& Yoshida, M. Proc. natn. Acad. Sci. U.S.A. 80, 3618-3622 (1983).

63. Kiyokawa, T., Yoshikura, H., Hattori, S., Seiki, M. \& Yoshida, M. Proc. natn. Acad. Sci U.S.A. 81, 6202-6206 (1984)

64. Sagata, N. et al Proc natn. Acad. Sci. U.S.A. 82, 677-681 (1985).

65. Schwartz, D. E., Tizard, R. \& Gilbert, W. Cell 32, 853-869 (1983).

\section{ETTERSTONATURE}

\section{Switching phenomena in a new 90-K superconductor}

\author{
P. H. Hor*, R. L. Meng*, C. W. Chu*, M. K. Wu†, \\ E. Zirngiebl‡, J. D. Thompson‡ \& C. Y. Huang§
}

* Department of Physics, University of Houston, Houston, Texas 77004, USA

$\dagger$ Department of Physics, University of Alabama, Huntsville, Alabama 35899, USA

‡ Los Alamos National Laboratory, Los Alamos, New Mexico 87545, USA

$\S$ Lockheed Palo Alto Research Laboratory, Palo Alto, California 94304-1191, USA

Recently, Wu et al. ${ }^{1}$ and Hor et al. ${ }^{2}$ have shown that $\mathrm{Y}_{1.2} \mathrm{Ba}_{0.8} \mathrm{CuO}_{4-\delta}$ is a superconductor with a superconducting onset temperature at $\sim 92 \mathrm{~K}$ as determined by their resistivity and a.c. susceptibility measurements. Because the magnetic properties are important in describing the nature of superconductivity, we have measured the d.c. magnetic moment of this material. Here we show that this material cooled in zero field or in a high field $\left(H_{\text {cool }}>\right.$
$90 \mathrm{G}$ ) is diamagnetic below $T_{\mathrm{cm}} \approx 90 \mathrm{~K}$, consistent with the previous measurements ${ }^{1,2}$. However, when the sample is cooled in a small field $(\leqslant 85 \mathrm{G})$, the magnetization, $M$, first becomes negative (diamagnetic) below $T_{\mathrm{cm}}$, but further cooling results in a jump of $M$ to a positive value at low temperature. We have also observed this switching by the application of an additional small field when the sample was cooled in a small field.

The $\mathrm{Y}_{1.2} \mathrm{Ba}_{0.8} \mathrm{CuO}_{4-\delta}$ sample was prepared as described in ref. 1. The $\mathrm{X}$-ray diffractograms reveal that the sample has multiple phases, devoid of the $\mathrm{K}_{2} \mathrm{NiF}_{4}$ structure. From the electrical resistance measurement, the superconducting onset temperature is $T_{\text {co }} \approx 94.5 \mathrm{~K}$ and the resistance becomes 'zero' below $T_{0}=92 \mathrm{~K}$ indicating that the sample is a superconductor with a rather narrow transition width. A Quantum Design superconducting quantum interference device (SQUID) magnetometer has been employed to measure the magnetization of the sample as a function of temperature and magnetic field. When the sample is cooled under zero field conditions, we have found that $M$ is diamagnetic below $T_{\mathrm{cm}}$ and the susceptibility below $\sim 25 \mathrm{~K}$ reaches $\sim 35 \%$ of that of perfect diamagnetism $(-1 / 4 \pi)$.

We have also measured $M$ when the sample is cooled in a field, $H_{\text {cool }}$. In Fig. 1 , the magnetization obtained at various 